\title{
The Induction of MicroRNA Targeting IRS-1 Is Involved in the Development of Insulin Resistance under Conditions of Mitochondrial Dysfunction in Hepatocytes
}

\author{
Hyun Su Ryu ${ }^{19}$, Seung-Yoon Park ${ }^{19}$, Duan $\mathrm{Ma}^{2}$, Jin Zhang ${ }^{2}$, Wan Lee ${ }^{1 *}$ \\ 1 Department of Biochemistry, Dongguk University College of Medicine, Kyungju, Korea, 2 Key Lab of Molecular Medicine, Shanghai Medical College, Institutes of \\ Biomedical Sciences, Fudan University, Shanghai, China
}

\begin{abstract}
Background: Mitochondrial dysfunction induces insulin resistance in myocytes via a reduction of insulin receptor substrate-1 (IRS-1) expression. However, the effect of mitochondrial dysfunction on insulin sensitivity is not understood well in hepatocytes. Although research has implicated the translational repression of target genes by endogenous non-coding microRNAs (miRNA) in the pathogenesis of various diseases, the identity and role of the miRNAs that are involved in the development of insulin resistance also remain largely unknown.
\end{abstract}

Methodology: To determine whether mitochondrial dysfunction induced by genetic or metabolic inhibition causes insulin resistance in hepatocytes, we analyzed the expression and insulin-stimulated phosphorylation of insulin signaling intermediates in SK-Hep1 hepatocytes. We used $q$ RT-PCR to measure cellular levels of selected miRNAs that are thought to target IRS-1 3' untranslated regions (3'UTR). Using overexpression of miR-126, we determined whether IRS-1-targeting miRNA causes insulin resistance in hepatocytes.

Principal Findings: Mitochondrial dysfunction resulting from genetic (mitochondrial DNA depletion) or metabolic inhibition (Rotenone or Antimycin A) induced insulin resistance in hepatocytes via a reduction in the expression of IRS-1 protein. In addition, we observed a significant up-regulation of several miRNAs presumed to target IRS-1 3'UTR in hepatocytes with mitochondrial dysfunction. Using reporter gene assay we confirmed that miR-126 directly targeted to IRS-1 3'UTR. Furthermore, the overexpression of miR-126 in hepatocytes caused a substantial reduction in IRS-1 protein expression, and a consequent impairment in insulin signaling.

Conclusions/Significance: We demonstrated that miR-126 was actively involved in the development of insulin resistance induced by mitochondrial dysfunction. These data provide novel insights into the molecular basis of insulin resistance, and implicate miRNA in the development of metabolic disease.

Citation: Ryu HS, Park S-Y, Ma D, Zhang J, Lee W (2011) The Induction of MicroRNA Targeting IRS-1 Is Involved in the Development of Insulin Resistance under Conditions of Mitochondrial Dysfunction in Hepatocytes. PLoS ONE 6(3): e17343. doi:10.1371/journal.pone.0017343

Editor: Jose Calbet, University of Las Palmas de Gran Canaria, Spain

Received August 13, 2010; Accepted January 31, 2011; Published March 25, 2011

Copyright: (c) 2011 Ryu et al. This is an open-access article distributed under the terms of the Creative Commons Attribution License, which permits unrestricted use, distribution, and reproduction in any medium, provided the original author and source are credited.

Funding: This study was supported by grant of the Korea Healthcare R\&D Project, Ministry of Health \& Welfare (A100370); 21C Frontier Functional Proteomics Project from Ministry of Education, Science and Technology (M108KM010008-08K1301-00810); National Research Foundation of Korea Grant (2009-006791), Republic of Korea. The funders had no role in study design, data collection and analysis, decision to publish, or preparation of the manuscript.

Competing Interests: The authors have declared that no competing interests exist.

*E-mail: wanlee@dongguk.ac.kr

9 These authors contributed equally to this work.

\section{Introduction}

Insulin resistance is defined as the decreased responsiveness of target tissues to ordinary levels of insulin and plays a central role in the development of metabolic disorders such as type 2 diabetes, hypertension, and dyslipidemia [1,2]. A number of studies have provided support for the hypothesis that genetic or functional impairments in mitochondria are involved in the development of insulin resistance [3,4]. Cellular oxidative capacity, which mostly depends on mitochondrial function, is directly correlated with insulin sensitivity in skeletal muscles $[5,6,7]$, and reduced mitochondria activity has been observed in patients with obesity and type 2 diabetes $[8,9,10]$. Although, several investigations demonstrated that skeletal muscle oxidative capacity and mitochondrial function are not a primary factor for insulin sensitivity in obese subjects $[11,12,13,14]$, emerging evidence support that mitochondrial dysfunction may play an important role in the pathogenesis of insulin resistance and type 2 diabetes [15,16,17]. Recently, it has been suggested that mitochondrial dysfunction induced by inhibitors of mitochondrial function or depletion of mitochondrial DNA (mtDNA) causes insulin resistance in myocytes through a reduction in the expression of insulin receptor substrate (IRS)-1, a protein with a pivotal role in the insulin signaling cascade $[18,19]$

IRS-1 is a key molecule in insulin signaling, and is involved in signal transduction between the insulin receptor and phosphoino- 
A

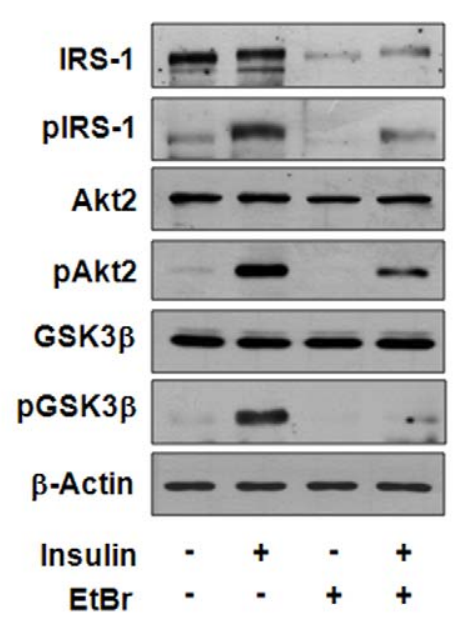

B

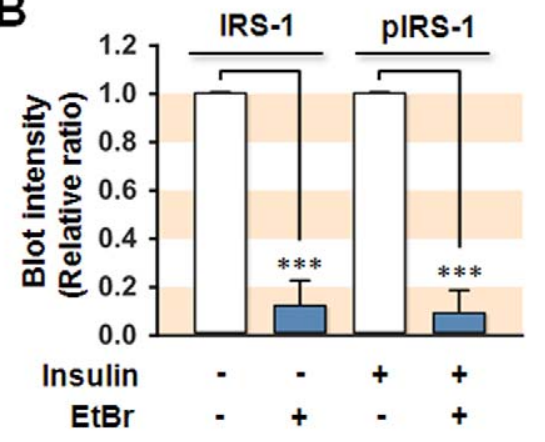

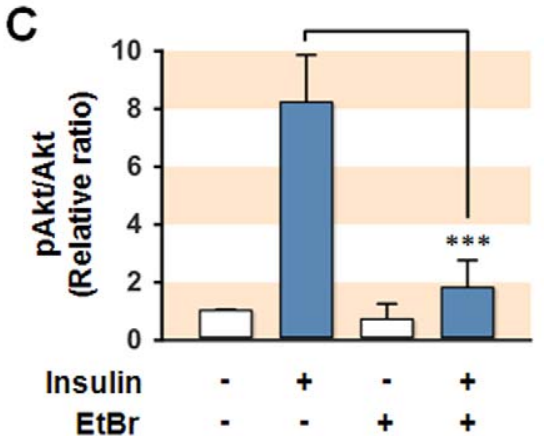

D
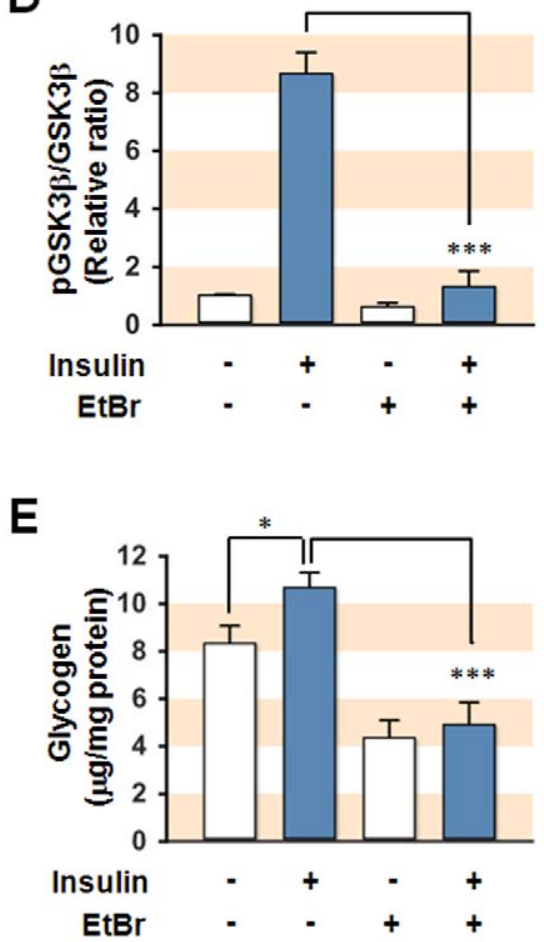

Figure 1. Effect of mtDNA depletion induced by EtBr on insulin signaling. SK-Hep1 hepatocytes depleted of mtDNA was prepared by EtBr treatment for 2 weeks. Cells were preincubated in the absence or presence of insulin (100 nM for $20 \mathrm{~min}$ ) and total cell lysates (15 $\mu \mathrm{g}$ protein) were subjected to $8 \%$ SDS-PAGE. (A) The expression (IRS-1, Akt2, and GSK3 $\beta$ ) and phosphorylation (pIRS-1, pAkt2, and pGSK3 $\beta$ ) of insulin signaling intermediates were analyzed by immunoblot. (B) The immunoblot intensities for IRS-1/ $\beta$-Actin (IRS-1) and insulin-stimulated pIRS-1/ $\beta$-Actin (pIRS-1) were quantified by densitometry and expressed in relative ratio where the intensity of normal control was set to one. (C-D) The immunoblot intensities for pAkt2/Akt2 and pGSK3B/GSK3 $\beta$ were quantified by densitometry and expressed in relative ratio. The intensity of normal control was set to one. (E) Cellular glycogen contents were analyzed by spectrophotometry $(570 \mathrm{~nm})$ with OxiRed probe. Values are expressed as means \pm SEM from at least five independent experiments. ${ }^{*}, \mathrm{P}<0.05 ;{ }^{* * *}, \mathrm{P}<0.001$.

doi:10.1371/journal.pone.0017343.g001

sitide 3-kinase (PI3K)[20]. Several lines of evidence suggest that a reduction in IRS-1 protein plays an important role in the development of insulin resistance and type 2 diabetes. IRS- 1 is decreased in skeletal muscle and liver of animal models established for insulin resistance and type 2 diabetes, such as $o b / o b$ mice [21,22] and Zucker fatty rats [23]. Studies have also reported decreased IRS-1 expression in the skeletal muscle of patients with diabetes, and concluded that this could represent a marker for the risk of insulin resistance [24,25,26,27]. However, the molecular mechanism underlying the reduction of IRS-1 expression in muscle and liver under conditions of mitochondrial dysfunction remains largely unknown.

MicroRNAs (miRNAs) are endogenous small non-coding RNAs that act as posttranscriptional regulators [28]. Mature miRNAs hybridize to partially complementary binding sites that are typically localized in the $3^{\prime}$ untranslated regions $\left(3^{\prime} \mathrm{UTR}\right)$ of target mRNAs [29]. This property allows a single miRNA sequence to have multiple target sites on various mRNAs. Upon binding, the miRNA initiates a pathway that either degrades the transcripts or suppresses its translation [29]. Although the target genes and biological functions of individual miRNAs remain largely unknown, it has been suggested that miRNAs have diverse functions in both normal and pathological states [28]. Recent research has established that unregulated miRNAs expression is implicated in defective insulin secretion, diabetic kidney and heart disease [30]. However, no previous study has investigated the involvement of miRNAs in metabolic disorders, in particular the development of insulin resistance. 

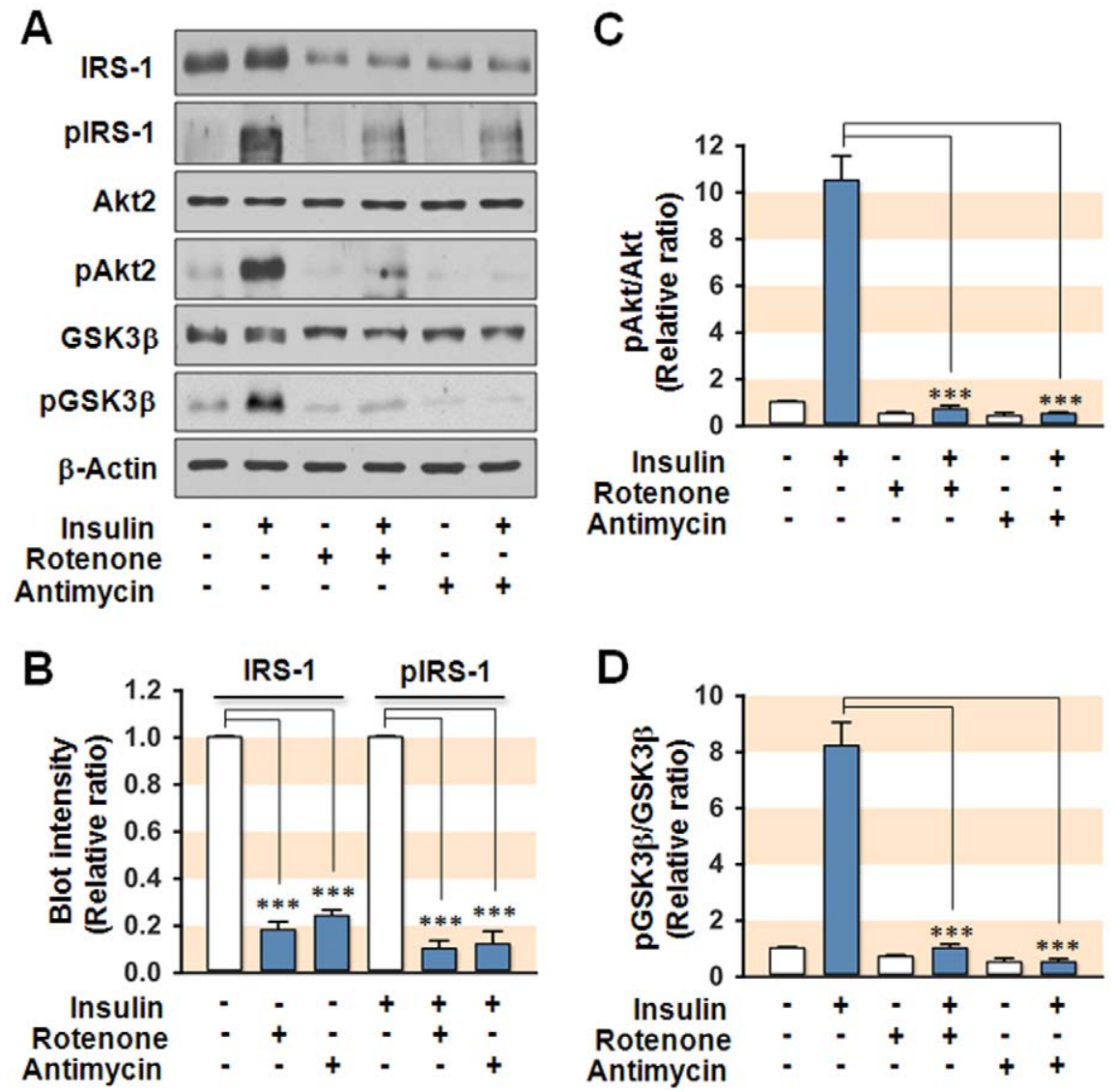

Figure 2. Effect of mitochondrial metabolic inhibitors on insulin signaling. For mitochondrial metabolic inhibition, hepatocytes were treated with Rotenone $(0.1 \mu \mathrm{M})$ or Antimycin A $(20 \mu \mathrm{M})$ for $18 \mathrm{~h}$. Cells were incubated in the absence or presence of insulin (100 $\mathrm{nM}$ for $20 \mathrm{~min})$ and total cell lysates (15 $\mu \mathrm{g}$ protein) were subjected to $8 \%$ SDS-PAGE. (A) The expression (IRS-1, Akt2, and GSK3 $\beta$ ) and phosphorylation (pIRS-1, pAkt2, and p GSK3 $\beta$ ) of insulin signaling intermediates were analyzed by immunoblot. (B) The immunoblot intensities for IRS-1/ $\beta$-Actin (IRS-1) and insulinstimulated pIRS-1/ $\beta$-Actin (pIRS-1) were quantified by densitometry and expressed in relative ratio where the intensity of normal control was set to one. (C-D) The immunoblot intensities for pAkt2/Akt2 and pGSK3 $\beta / G S K 3 \beta$ were quantified by densitometry and expressed in relative ratio. The intensity of normal control was set to one. Values are expressed as means \pm SEM from at least five independent experiments.***, $\mathrm{P}<0.001$. doi:10.1371/journal.pone.0017343.g002

In the present study, we demonstrated that mitochondrial dysfunction resulting from genetic or metabolic inhibition provoked insulin resistance via a decrease in the expression of IRS-1. Furthermore, we found that mitochondrial dysfunction induced the expression of several miRNAs thought to target the IRS-1 3'UTR and that miR-126 was actively involved in the development of insulin resistance. Our findings in hepatocytes reveal a novel mechanism for the development of insulin resistance by providing the first direct evidence that miR-126 mediates the repression of IRS-1 expression in mitochondrial dysfunction.

\section{Results}

Hepatocytes depleted of mtDNA were prepared by exposing SK-Hepl hepatocytes to a low dose of ethidium bromide $(\mathrm{EtBr}$, $0.2 \mu \mathrm{g} / \mathrm{ml}$ ) for two weeks, as described previously for myocytes [18]. As shown in Figure S1, treatment with $\mathrm{EtBr}$ depleted mtDNA without altering nuclear DNA, and provoked mitochondrial dysfunction. To determine whether mitochondrial dysfunction induced by mtDNA depletion causes insulin resistance in hepatocytes, we analyzed the expression and insulin-stimulated phosphorylation of insulin signaling intermediates, such as IRS-1, Akt2, and glycogen synthase kinase-3 $\beta$ (GSK3 $\beta$ ), in the mtDNAdepleted SK-Hepl hepatocytes. We observed about $90 \%$ reduction in the expression of IRS-1 in the cells depleted of mtDNA by EtBr treatment (Figure $1 A-B$ ). As expected, mtDNA depletion significantly reduced the phosphorylation of IRS-1 and its substrate, Akt2 (Figure $1 B-C$ ). We also found a significant reduction in the insulin-stimulated phosphorylation of GSK3 $\beta$, a substrate of Akt2, in the mtDNA-depleted hepatocytes compared to control hepatocytes (Figure $1 D$ ). In addition, cellular glycogen contents in the presence or absence of insulin were analyzed in the control and mtDNA-depleted cells (Figure $1 E$ ). As we expected, insulin significantly increased glycogen contents in control cells. However, the depletion of mtDNA by EtBr significantly reduced basal glycogen content as compared to control. Moreover, insulinstimulated glycogen synthesis was almost completely disappeared in mtDNA-depleted hepatocytes (Figure $1 E$ ). These findings clearly indicate that mitochondrial dysfunction resulting from mtDNA depletion induces the development of insulin resistance in hepatocytes through a reduction in the expression of IRS-1.

We then investigated whether mitochondrial dysfunction resulting from metabolic inhibitors, such as Rotenone (an inhibitor of electron transfer from complex I to ubiquinone) and Antimycin A (an inhibitor of complex III), causes impairment of insulin signaling in SK-Hepl hepatocytes. We observed an almost complete loss of functional mitochondria in the hepatocytes treated with Rotenone or Antimycin A (Figure S2), whereas the 
A

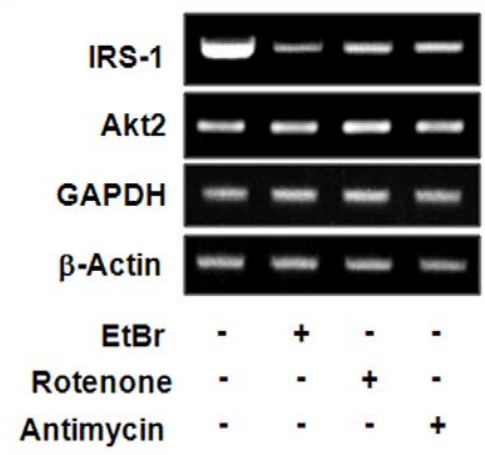

B

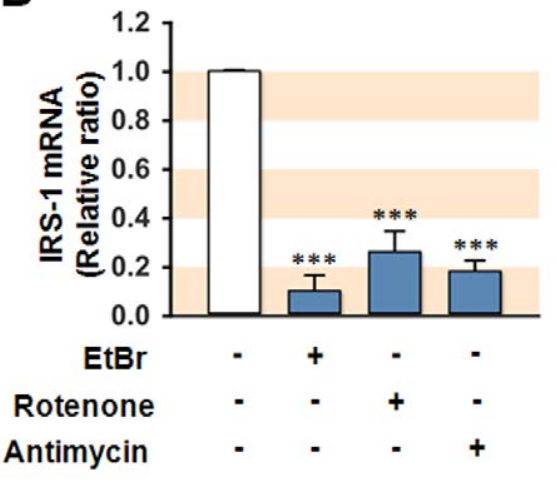

Figure 3. Transcriptional inhibition of IRS-1 in hepatocytes with dysfunctional mitochondria. SK-Hep1 hepatocytes were treated with vehicle, $\mathrm{EtBr}(0.2 \mu \mathrm{g} / \mathrm{ml}, 2$ week), Rotenone $(0.1 \mu \mathrm{M}$, $18 \mathrm{~h})$, or Antimycin $\mathrm{A}(20 \mu \mathrm{M}, 18 \mathrm{~h})$, and the expression levels of IRS-1, Akt2, GAPDH, and $\beta$-Actin were measured by (A) RT-PCR and (B) qRT$P C R$. The fold changes are expressed in relative ratio where the intensity of $\beta$-Actin was set to one. All results represent mean \pm SEM from three independent experiments. ${ }^{* *}, \mathrm{P}<0.001$ vs Control.

doi:10.1371/journal.pone.0017343.g003

level of mtDNA and its transcripts were not affected by inhibitors (data not shown). Interestingly, treatment with Rotenone or Antimycin A caused a significant reduction in the expression of IRS-1, whereas the expression of both Akt2 and GSK $3 \beta$ remained unaffected as compared to control (Figure $2 A-B$ ). Both inhibitors caused a significant reduction in the insulin-stimulated phosphorylation of IRS-1, Akt2, and GSK3 $\beta$ (Figure $2 B-D$ ). These findings suggest that mitochondrial dysfunction secondary to metabolic inhibition induces the development of insulin resistance in hepatocytes by decreasing the expression of IRS-1.

Although mitochondrial dysfunction provoked a substantial decline of IRS- 1 mRNA as shown in Figure $3 A-B$, posttranscriptional repression of IRS-1 should not be excluded. Therefore, to determine whether miRNA induces repression of IRS-1 expression posttranscriptionally, we selected several miRNAs targeting IRS-1 and measured cellular levels of these miRNAs in hepatocytes with dysfunctional mitochondria. Although mitochondrial dysfunction causes numerous changes in the expression of miRNAs in SK-Hepl cells, we initially selected miRNAs presumably targeting a segment of IRS-1 3'UTR (within 500 nucleotides from the stop codon) by TargetScan, MiBase, and PicTar analysis. These analyses revealed that about 70 miRNAs presumably targeting this region of IRS-1 3'UTR. Subsequently, as shown in Table $\mathrm{Sl}$, we selected 11 miRNAs for the next experiments according to its targeting score to IRS-1 3'UTR, as well as quality and quantity of the results from $q$ RT-PCR analysis. Although it has been reported that miR-145 targets IRS-1 in colon cancer cells [31], we excluded miR-145 in this study because of its low expression level. Interestingly, mtDNA depletion significantly (more than 2-fold, $\mathrm{P}<0.05$ ) increased the levels of several of these miRNAs including miR-27a, miR-27b, miR-30e, and miR-126, whereas the levels of other selected miRNAs remained unaffected (Figure 4A). In experiments in which mitochondrial dysfunction was induced by Rotenone (Figure $4 B$ ) or Antimycin A (Figure 4C), a significant increase in the levels of all of the selected miRNAs was observed, with the exception of miR-7. Tunicamycin is well known to induce endoplasmic reticulumn (ER) stress in various cells including SK-Hepl hepatocytes. Although treatment with tunicamycin $(2 \mu \mathrm{g} / \mathrm{ml})$ induced ER stress assessed by phosphorylation of eIF2 $\alpha$ (Figure S3), tunicamycin resulted in no significant change in the levels of any of the 11 selected miRNAs (Figure 4D) or the expression of IRS-1 (Figure S3). In addition, the levels of miRNAs such as miR-137, miR-663, miR-1231, miR-1246, and miR-1287, which are not targeting IRS-1 3'UTR, were not affected by mitochondrial dysfunction (data not shown). This result indicates that mitochondrial dysfunction increases the level of several miRNAs that are predicted to target IRS-1 3'UTR, thus potentially implicating miRNAs in the development of insulin resistance.

Research findings have suggested that these up-regulated miRNAs may be translational repressors of IRS-1 expression, and that they may be involved in the development of insulin resistance. Although we selected miRNAs that are thought to target IRS-1 3'UTR, most of them have not yet been validated for whether they actually repress IRS-1 expression. Recently, Zhang et al. [32] found that miR-126 inhibited cell cycle progression in cancer cells via the targeting of IRS-1 $3^{\prime} \mathrm{UTR}$. We therefore selected miR-126 for further investigation of its functional relevance in the development of insulin resistance. To determine whether miR-126 directly regulates the expression of IRS-1 in SKHepl hepatocytes, we set up an assay in which 414 nt $3^{\prime}$ UTR of the IRS-1 gene, which contains miR-126 binding site (Figure 5A$B$ ), was inserted downstream of a luciferase open reading frame (IRS1 3Uwt). A mutated 3'UTR of the IRS-1 gene (IRS1 3Umut), which lacks miR-126 binding site, was used as a control (Figure 5B). Plasmid DNA of each pGL3-promoter-based 3'UTR reporter (IRS1-3Uwt or IRS1-3Umut) was cotransfected with the indicated concentration of empty plasmid (pSilence6.1) or miR126 expression plasmid into SK-Hepl hepatocytes. We found that luciferase activity with IRS1-3Uwt was significantly inhibited by miR-126 in a dose-dependent manner, whereas luciferase activity with IRS1-3Umut was not (Figure $5 C$ ). These data suggest that miR-126 directly targets IRS-1 3'UTR and suppresses IRS-1 expression at the posttranscriptional level.

To confirm that IRS-1 is a target gene of miR-126 in hepatocytes, we transfected SK-Hepl cells with empty plasmid or miR-126 expression plasmid, and investigated whether induction of miR-126 directly represses IRS-1 expression. As shown in Figure 6, the overexpression of miR-126 resulted in a substantial reduction in the level of IRS-1 protein with no apparent change in the level of IRS-1 mRNA, thereby indicating the posttranscriptional repression of IRS-1 expression by miR-126. However, the protein levels of Akt2 and GSK3 $\beta$ remained unaffected (Figure $6 A-B$ ). We also examined whether miR-126 could induce posttranslational degradation of IRS-1 in SK-Hepl cells (Figure 6C). Cycloheximide, an inhibitor of peptidyl transferase in eukaryotic ribosome, is used for the analysis of IRS-1 degradation rate by blocking de novo synthesis of proteins. 
A

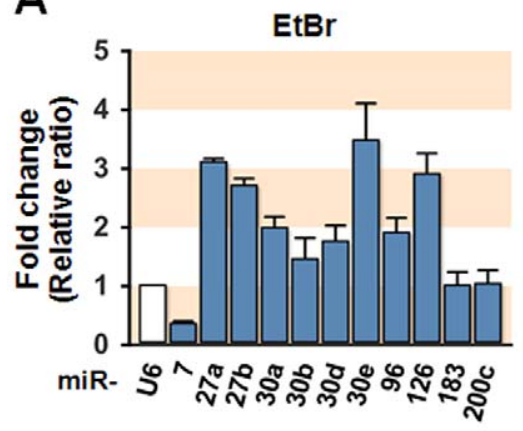

B

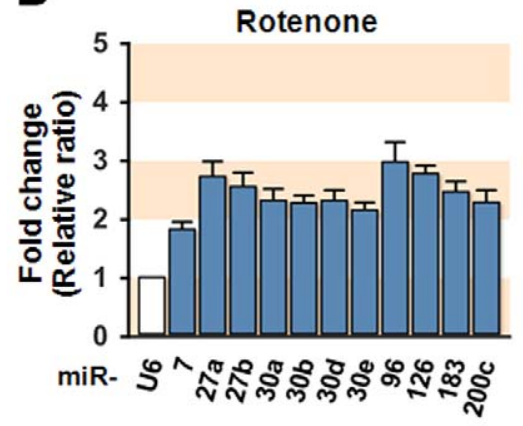

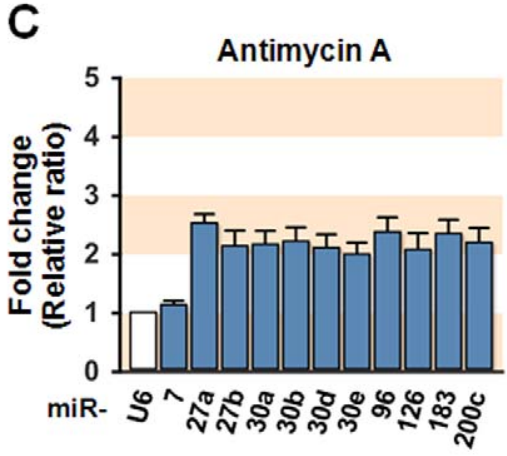

D

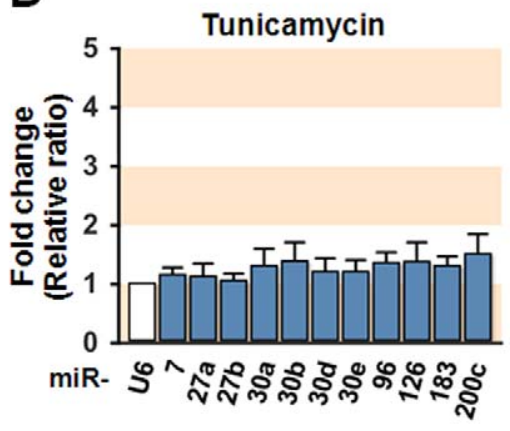

Figure 4. Expression of miRNAs presumably targeting IRS-1 3'UTR. SK-Hep1 hepatocytes were treated with (A) EtBr (0.2 $\mu \mathrm{g} / \mathrm{ml}, 2 \mathrm{week})$, (B) Rotenone $(0.1 \mu \mathrm{M}, 18 \mathrm{~h}),(\mathbf{C})$ Antimycin A $(20 \mu \mathrm{M}, 18 \mathrm{~h})$, or (D) Tunicamycin $(2 \mu \mathrm{g} / \mathrm{ml}, 18 \mathrm{~h})$, and the expression levels of miRNAs that predicted to target IRS-1 3'UTR were measured by qRT-PCR. The fold changes are expressed in relative ratio where U6 intensity set to one. doi:10.1371/journal.pone.0017343.g004

Although overexpression of miR-126 significantly reduced expression of IRS-1, the degradation rate of IRS-1 was not affected by miR-126. Therefore, it is suggested that the forced expression of miR-126 in hepatocytes does not affect the expression of IRS-1 posttranslationally.

Next, we analyzed expression and insulin-stimulated phosphorylation of insulin signaling intermediates in the hepatocytes transfected with miR-126 expression plasmid (Figure 7). The expression of IRS-1 was significantly reduced by $75 \%$ after miR126 overexpression, whereas expression of Akt2 and GSK $3 \beta$ was not affected. As expected, the overexpression of miR-126 significantly reduced insulin-stimulated phosphorylation of IRS-1 and its downstream kinases, Akt2 and GSK $3 \beta$ in hepatocytes, and this effect was mainly due to a reduction in the expression of IRS-1 (Figure $7 B-D$ ). In addition, we determined how the overexpression of miR-126 affects cellular glycogen contents in the presence or absence of insulin (Figure $7 E$ ). In control cells, insulin significantly increased glycogen contents in hepatocytes. However, glycogen content in miR-126-overexpressed hepatocytes was significantly reduced as compared to control. Moreover, induction of miR-126 abolished insulin-stimulated glycogen synthesis (Figure $7 E$ ). Taken together, these data clearly indicate that the induction of miR-126 causes insulin resistance in hepatocytes through a reduction in the expression of IRS-1.

\section{Discussion}

Mitochondrial dysfunction is associated with the development of insulin resistance and diabetes $[3,4,16]$. In the present study, we demonstrated that mitochondrial dysfunction resulting from genetic or metabolic inhibition induced the development of insulin resistance in hepatocytes via a reduction of IRS-1 expression. This result is in accordance with previous findings in myocytes that a reduced expression of IRS-1 is involved in the development of insulin resistance $[18,19]$. Recent work in myocytes has revealed that mitochondrial retrograde signals inhibit the expression of IRS- 1 at the transcriptional level by activating c-Jun N-terminal kinase (JNK) and p38 mitogen-activated protein kinase (MAPK), which in turn up-regulates an IRS-1 transcriptional suppressor, ATF3 [19]. Although the regulation of IRS-1 expression occurs at the transcriptional level under conditions of mitochondrial dysfunction, posttranscriptional regulation also occurs. For example, mitochondrial dysfunction activates certain stress kinases, such as IкB kinase $\beta$ and JNK. These are well-known serine kinases that phosphorylate IRS-1 at serine residues, leading to decreased metabolic signaling by increasing IRS-1 degradation and serine phosphorylation [16]. Although coordinated alteration in expression of skeletal muscle miRNAs relates to insulin resistance in diabetes [33], the involvement of miRNAs in posttranscriptional repression of IRS-1 are largely unknown, especially in mitochondrial dysfunction,

The fact that no previous studies have investigated the role of miRNAs in insulin signaling accounts for the lack of research into their potential as therapeutic targets for metabolic disease. We hypothesized that certain miRNAs may participate in the development of insulin resistance induced by mitochondrial dysfunction. We observed a significant increase in the expression of several presumed IRS-1-3'UTR-targeting miRNAs in hepatocytes with dysfunctional mitochondria. In particular, we found that ectopic expression of miR-126, an induced IRS-1-targeting miRNAs, represses translation of IRS-1 without altering the mRNA level of IRS-1, which subsequently leads to insulin resistance in hepatocytes. Expression of miR-126 is decreased in various cancer cells, and it has previously been regarded as a cell 
A

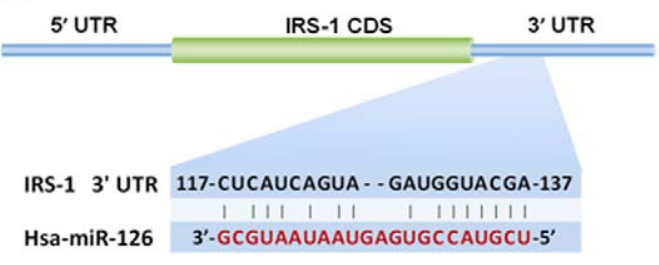

B

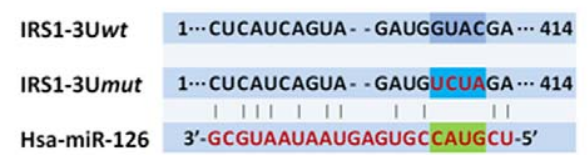

C

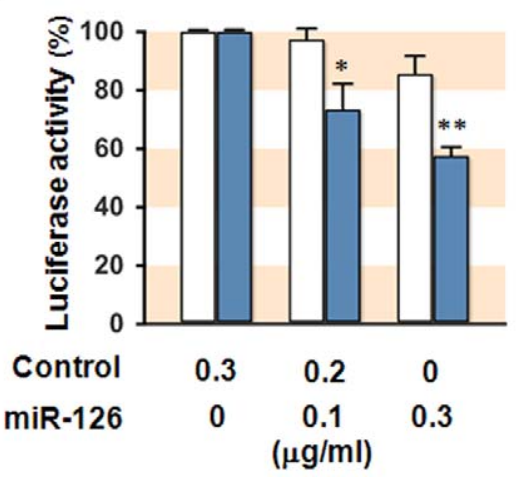

Figure 5. Targeting site of miR-126 in the $3^{\prime}$ UTR of IRS-1 and measurement of its binding by reporter gene assay. (A) Seed sequence of miR-126 is predicted to target IRS-1 3'UTR. (B) For reporter gene assay, 414 nt-long $3^{\prime}$ UTR of the IRS-1 gene was inserted downstream of a luciferase open reading frame (IRS1-3Uwt). As a control, a mutated 3'UTR of IRS-1 gene (IRS1 3Umut) lacking the miR126 binding site was used. (C) IRS1-3Uwt (closed column) or IRS13Umut (open column) construct was cotransfected with the indicated concentration of empty plasmid (Control) or miR-126 expression plasmid (miR-126) into SK-Hep1 hepatocytes. Reporter gene assay were performed using a Dual-luciferase assays kit as described in Methods. The relative luciferase activities were plotted against that of control, which is set as $100 \%$. Values are expressed as means \pm SEM from four independent experiments. ${ }^{*}, \mathrm{P}<0.05{ }^{* *}, \mathrm{P}<0.01$ vs IRS13Umut.

doi:10.1371/journal.pone.0017343.g005

growth suppressor that acts on IRS-1 [32], HOXA9 [34] and p85 $\beta$ [35]. In the present study, we have unveiled a new role of miR-126 as a metabolic regulator. Further, several other miRNAs predicted to target the IRS-1 3'UTR were induced by mitochondrial dysfunction in a similar fashion to miR-126, it is still open questions whether these miRNAs directly target the $3^{\prime} \mathrm{UTR}$ and repress IRS-1 expression. In addition, the expression of miRNA target genes can be modulated by altering the identity or the concentration of the miRNAs within cells [36]. Therefore, the combinational binding of different miRNAs on non-overlapping binding sites of IRS-1 3'UTR may synergistically potentiate the miRNA-induced repression of IRS-1. The discovery that miR-126 regulates insulin signaling provides novel insights into the molecular basis underlying mitochondrial dysfunction-derived pathogenesis of insulin resistance, and implicates miRNAs in metabolic diseases.

Here, we propose a possible mechanism for how miRNA participates in the development of insulin resistance under conditions of mitochondrial dysfunction. Mitochondrial dysfunction results in the generation of various retrograde signals to the nucleus, as described initially in myocytes [37]. These retrograde signals activate several kinases and transcriptional factors, resulting in the suppression of IRS-1 transcription and induction of IRS-1 degradation [19]. However, the results of present study suggest that some of the transcriptional factors that are affected by this retrograde signaling may activate the production and processing of miRNAs targeting the IRS-1 3'UTR, thereby leading to IRS-1 posttranscriptional repression and subsequent insulin resistance. Further analysis of which metabolic signals and transcriptional factors are involved and how they work in miRNA-mediated insulin resistance will be the next step of research.

In conclusion, we have demonstrated that mitochondrial dysfunction induced by mtDNA depletion or metabolic inhibition causes the development of insulin resistance in hepatocytes through a reduction in the expression of IRS- 1 . We found that mitochondrial dysfunction significantly up-regulated the expression of several miRNAs that are thought to target IRS-1. We confirmed that miR-126 is a crucial inhibitory factor in insulin signal transduction under conditions of mitochondrial dysfunction. Consequently, these data provide a novel mechanism for the development of insulin resistance, and serve as a foundation for further studies designed to explore the functional relevance of miRNAs in diabetes and metabolic syndrome.

\section{Materials and Methods}

Cell culture and induction of mitochondrial dysfunction

SK-Hepl hepatocytes (ATCG HTB-52) were cultured in Dulbecco's Modified Eagle Medium (DMEM) with 10\% fetal bovine serum (FBS). For mtDNA depletion, cells were incubated with $\operatorname{EtBr}(0.2 \mu \mathrm{g} / \mathrm{ml})$ and uridine $(50 \mu \mathrm{g} / \mathrm{ml})$ for 2 weeks in DMEM with $10 \%$ FBS. Under these experimental conditions, mtDNA was depleted to $<10 \%$ of normal. For mitochondrial metabolic inhibition, cells were treated with Rotenone $(0.1 \mu \mathrm{M})$ or Antimycin A $(20 \mu \mathrm{M})$ for $18 \mathrm{~h}$. Hepatocytes were deprived of serum for $5 \mathrm{~h}$ prior to all experimental analysis.

\section{Genomic DNA extraction and polymerase chain reaction (PCR)}

Total cellular DNA was extracted according to the manufacturer's instructions by using DNeasy Tissue kit (Qiagen). PGR and $q$ RT-PCR was performed in LightCycler ${ }^{\circledR} 480$ (Roche-Applied Science) using SYBR-Green PCR Master Mix according to manufacturer's instructions (Qiagen) with the specific primers listed in Table S1.

\section{Staining of functional mitochondria}

SK-Hepl hepatocytes were incubated with the active mitochondrial-specific fluorescent dye, MitoTracker Orange CM$\mathrm{H}_{2}$ TMRos (Molecular Probes, Eugene, OR), for $15 \mathrm{~min}$ at $37^{\circ} \mathrm{C}$ and washed three times with cold PBS. The cells were visualized and photographed in a fluorescence microscope (Leica, Germany). The fluorescent intensity reflects the integrity of mitochondrial function [38]. 
A
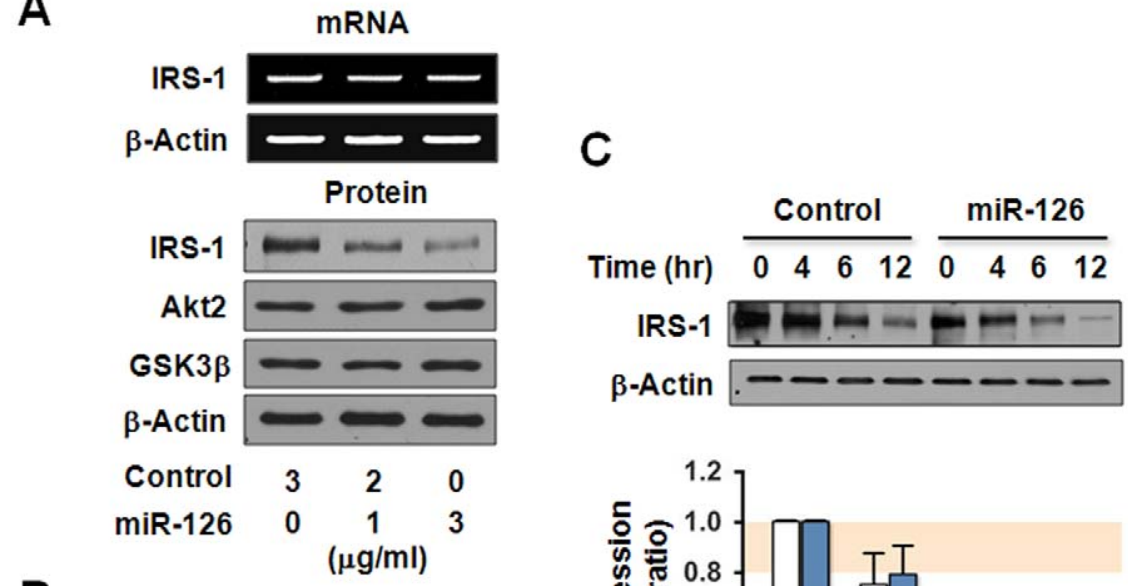

B
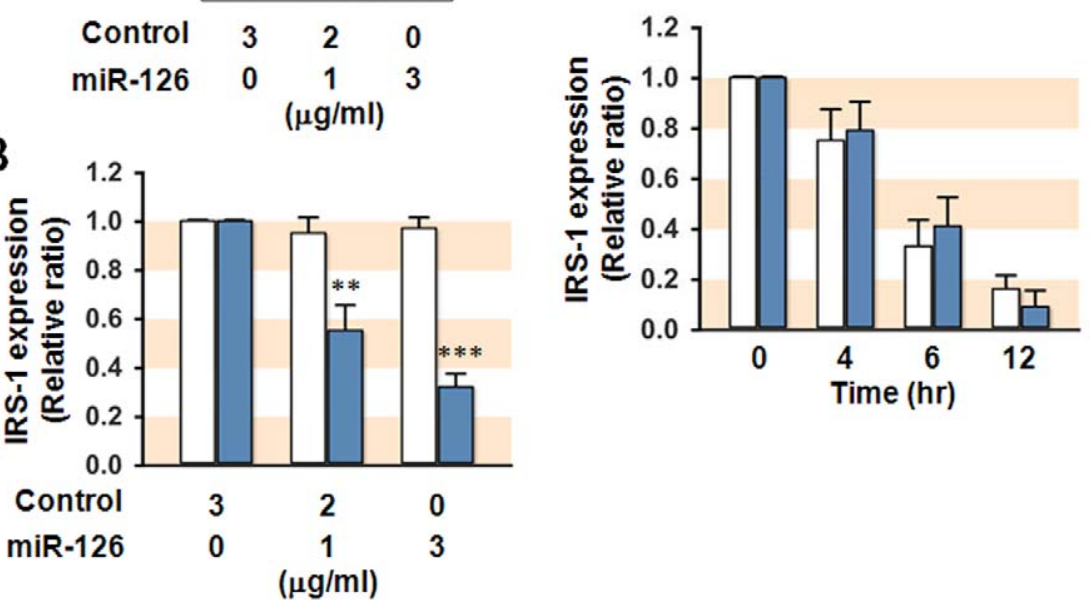

Figure 6. Effect of miR-126 on expression of IRS-1. SK-Hep1 hepatocytes were transfected with the indicated concentration of empty plasmid (Control) or miR-126 expression plasmid (miR-126). (A) mRNA level was analyzed $24 \mathrm{~h}$ of transfection, whereas immunoblot analysis was conducted after $72 \mathrm{~h}$ of transfection. These are representative results from five independent experiments. (B) Expressions of IRS-1 were analyzed by $q R T-P C R$ (open column) and immunoblotting (closed column). The mRNA and protein of IRS-1 were expressed in relative ratio, where the intensity of empty plasmid alone was set to one. (C) SK-Hep1 hepatocytes were transfected with $3 \mu \mathrm{g} / \mathrm{ml}$ of empty plasmid (Control, open column) or miR-126 expression plasmid (miR-126, closed column). After $60 \mathrm{~h}$ of transfection, cells were treated with cycloheximide $(50 \mu \mathrm{g} / \mathrm{ml}$ for $12 \mathrm{~h})$ and total cell lysates ( $15 \mu \mathrm{g}$ protein) were subjected to $8 \%$ SDS-PAGE. The protein levels of IRS-1 were analyzed by densitometry and expressed in relative ratio, where the intensity of $0 \mathrm{~h}$ was set to one. Values are expressed as means \pm SEM from at least five independent experiments. ${ }^{* *}, \mathrm{P}<0.01 ;{ }^{* * *}, \mathrm{P}<0.001$. doi:10.1371/journal.pone.0017343.g006

\section{Measurement of cellular ATP levels}

The cellular ATP was measured by using a somatic cell ATP assay kit (Sigma, Louis, MO) as described in the manufacturer's procedure.

RNA preparation and quantitative real-time RT-PCR (qRTPCR)

Total cellular RNA was extracted using miRNeasy Mini kit (Qiagen, Valencia, CA). For $q$ RT-PCR, total RNAs were reverse transcribed into cDNAs with miScript Reverse Transcription kit (Qiagen) and amplified with specific primers (Bionics, Seoul, Korea) listed in Table S1. $q$ RT-PCR was carried out in LightCycler ${ }^{\circledR} 480$ (Roche-Applied Science, Mannheim, Germany) using SYBR-Green PCR Master Mix according to manufacturer's instructions (Qiagen).

\section{Overexpression of miR-126 and reporter gene assay}

Plasmids (pSilencer4.1CMV-puro, Ambion) expressing miR126 pre-miRNA (flanking upstream and downstream 30-50 nt) for overexpression of miR-126 was produced as described previously [32] and transfected to SK-Hepl hepatocytes with Lipofectamine 2000. For luciferase assay, DNA fragments (414 nt) of IRS-1 3'UTR containing predicted miR-126 binding site (IRS1-3Uwt) were cloned into the pGL3-promoter plasmid (Promega) and the mir-126 binding sites were replaced with an 4 nt fragment to produce mutated 3'UTR pGL3 report plasmids (IRS1-3Umut) as described [32]. Luciferase assays were performed with the Dual luciferase reporter system (Promega) in accordance with manufacturer's instructions. Briefly, SK-Hepl cells were plated the day before transfection onto 12 -well plates and grown to an approximate $70 \%$ confluence. The cells were cotransfected with IRS1-3Uwt or 3Umut and miR-126 expression plasmid together with pRL-SV40 for constitutive expression of Renilla luciferase as an internal control. At $48 \mathrm{~h}$ posttransfection, the cells were lysed and subjected to the measurement of the firefly and Renilla luciferase activities on a Lumat LB 9507 luminometer (EGG, Stuttgart, Germany). The ratio of firefly luciferase activity to Renilla luciferase activity was presented in arbitrary units as the relative luciferase activities.

\section{Glycogen Assay}

Glycogen contents in the cultured cells were analyzed with Glycogen Assay Kit (BioVision, Mountain View, CA) by spectrophotometry $(570 \mathrm{~nm})$ with OxiRed probe according to manufacturer's suggestion.

\section{Gel Electrophoresis and Immunoblotting}

Cell lysates solubilized in Laemmli solution were subjected to SDS-PAGE on 8 or $10 \%$ resolving gels and immunoblotting as 

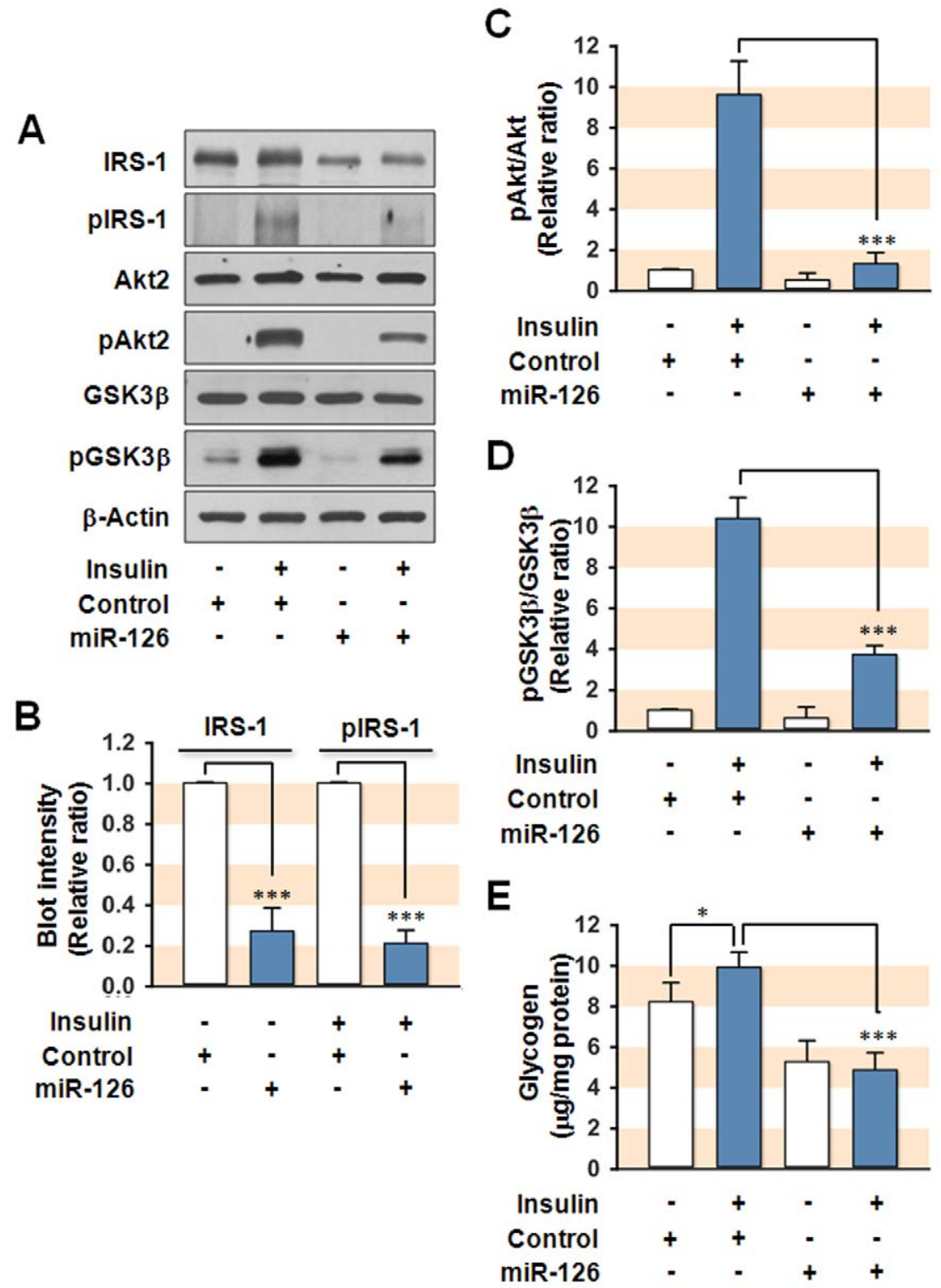

Figure 7. Effect of miR-126 on insulin signaling. SK-Hep 1 hepatocytes were transfected with $3 \mu \mathrm{g} / \mathrm{ml}$ of empty plasmid (Control) or miR-126 expression plasmid (miR-126). After $72 \mathrm{~h}$ of transfection, cells were preincubated in the absence or presence of insulin (100 nM for 20 min) and total cell lysates (15 $\mu \mathrm{g}$ protein) were subjected to $8 \%$ SDS-PAGE. (A) The expression (IRS-1, Akt2, and GSK3 $\beta$ ) and phosphorylation (pIRS-1, pAkt2, and pGSK3 $\beta$ ) levels of insulin signaling intermediates were analyzed by immunoblot. (B) The immunoblot intensities for IRS-1/ $\beta$-Actin (IRS-1) and insulinstimulated pIRS-1/ $\beta$-Actin (pIRS-1) were quantified by densitometry and expressed in relative ratio, where the intensity of normal control was set to one. (C-D) The immunoblot intensities for pAkt2/Akt2 and pGSK3 $3 / G S K 3 \beta$ were quantified by densitometry and expressed in relative ratio. The intensity of normal control was set to one. (E) Cellular glycogen contents were analyzed by spectrophotometry (570 nm) with OxiRed probe. Values are expressed as means \pm SEM from at least five independent experiments. ${ }^{*}, \mathrm{P}<0.05 ;{ }^{* *}, \mathrm{P}<0.001$.

doi:10.1371/journal.pone.0017343.g007

described [39]. Antibody against IRS-1 was purchased from Upstate (Lake Placid, NY) and antibody against phosphor IRS-1 was from Invitrogen (Carlsbad, CA). All other antibodies were obtained from Cell Signaling (Beverly, MA). Proteins were visualized using enhanced chemiluminescent substrate kit (NEN Life Science Products). Immunoblot intensities were quantitated by densitometry using an analytical scanning system (Molecular Dynamics Inc., Sunnyvale, CA).

\section{Database and Statistical analysis}

We computationally screened miRNAs targeting IRS-1 3'UTR with TargetScan (http://www.targetscan.org/index.html) and
miRBase (http://microrna.sanger.ac.uk). Results were expressed as the mean \pm SEM. Where applicable, the significance of difference was analyzed using Student's $t$ test for unpaired data.

\section{Supporting Information}

Figure S1 Mitochondrial dysfunction induced by EtBr. (A) Genomic DNA and mRNA were isolated from control or EtBrtreated SK-Hepl hepatocytes, and mtDNA-encoded genes such as cytochrome c oxidase subunit I (COX-I) and subunit II (COX-II), and nuclear DNA-encoded gene such as cytochrome c oxidase subunit IV (COX-IV) were amplified by RT-PGR and $q$ RT-PGR. 
(B) The relative values were expressed in arbitrary units where the intensity of control was set to one. (C) Control and EtBr-treated SKHepl hepatocytes were stained with the active mitochondrialspecific fluorescent dye, MitoTracker Orange CM- $\mathrm{H}_{2}$ TMRos, as described in Methods. Magnification is $\sim \mathrm{X} 400$. (D) Total cellular ATP levels were measured by the luciferin/luciferase assay. ATP content was expressed in arbitrary units where the ATP content from control cells was set to one. All results represent mean \pm SEM from three independent experiments. **, $\mathrm{P}<0.01$; ***, $\mathrm{P}<0.001$. Results: As shown in Figure S1 (A and B), cytochrome oxidase subunits I (COX-I) and II (COX-II), both encoded only in mtDNA, were hardly amplified from the genomic DNA and cDNA of the cells treated with $\mathrm{EtBr}$ for 2 weeks. In contrast, nuclear DNAencoded genes such as COX-IV and $\beta$-actin were detected at similar levels in both control and EtBr-treated hepatocytes, indicating that prolonged treatment with $\mathrm{EtBr}$ depleted the cellular contents of mtDNA and its transcripts without altering the nuclear DNA replication. Next, we measured mitochondrial function with the active mitochondrial-specific fluorescent dye, MitoTracker Orange CM-H $\mathrm{H}_{2}$ TMRos $[40,41,42,43]$. Since this Mitotracker localizes in mitochondria in a $\Delta \Psi_{\mathrm{m}}$-dependent manner, the fluorescent intensity from living cells thoroughly reflects the integrity of mitochondrial function. The depletion of mtDNA induced by $\mathrm{EtBr}$ treatment drastically reduced functional mitochondria in the cell $(\mathrm{C})$. In addition, the depletion of mtDNA significantly reduced total cellular ATP level as compared to control cells (D). These data clearly indicates that cellular treatment with EtBr depleted mtDNA without altering nuclear DNA and provoked mitochondrial dysfunction in SK-Hepl hepatocytes.

(TIF)

\section{References}

1. Kahn SE, Hull RL, Utzschneider KM (2006) Mechanisms linking obesity to insulin resistance and type 2 diabetes. Nature 444: 840-846.

2. Petersen KF, Shulman GI (2006) Etiology of insulin resistance. Am J Med 119: S10-16.

3. Marx J (2004) Medicine. Metabolic defects tied to mitochondrial gene. Science 306: 592-593.

4. Maassen JA, Leen MtH, Van Essen E, Heine RJ, Nijpels G, et al. (2004) Mitochondrial diabetes: molecular mechanisms and clinical presentation. Diabetes 53(Suppl 1): S103-109.

5. Kelley DE, Goodpaster BH (2001) Skeletal muscle triglyceride. An aspect of regional adiposity and insulin resistance. Diabetes Care 24: 933-941.

6. Simoneau JA, Colberg SR, Thaete FL, Kelley DE (1995) Skeletal muscle glycolytic and oxidative enzyme capacities are determinants of insulin sensitivity and muscle composition in obese women. Faseb J 9: 273-278.

7. Simoneau JA, Kelley DE (1997) Altered glycolytic and oxidative capacities of skeletal muscle contribute to insulin resistance in NIDDM. J Appl Physiol 83: $166-171$.

8. Kelley DE, He J, Menshikova EV, Ritov VB (2002) Dysfunction of mitochondria in human skeletal muscle in type 2 diabetes. Diabetes 51: 2944-2950.

9. Petersen KF, Dufour S, Befroy D, Garcia R, Shulman GI (2004) Impaired mitochondrial activity in the insulin-resistant offspring of patients with type 2 diabetes. N Engl J Med 350: 664-671.

10. Szendroedi J, Schmid AI, Chmelik M, Toth C, Brehm A, et al. (2007) Muscle mitochondrial ATP synthesis and glucose transport/phosphorylation in type 2 diabetes. PLoS Med 4: e154.

11. Schenk S, Harber MP, Shrivastava CR, Burant CF, Horowitz JF (2009) Improved insulin sensitivity after weight loss and exercise training is mediated by a reduction in plasma fatty acid mobilization, not enhanced oxidative capacity. J Physiol 587: 4949-4961.

12. Holloszy JO (2009) Skeletal muscle "mitochondrial deficiency" does not mediate insulin resistance. Am J Clin Nutr 89: 463S-466S.

13. Berggren JR, Boyle KE, Chapman WH, Houmard JA (2008) Skeletal muscle lipid oxidation and obesity: influence of weight loss and exercise. Am J Physiol Endocrinol Metab 294: E726-732.

14. Toledo FG, Menshikova EV, Azuma K, Radikova Z, Kelley CA, et al. (2008) Mitochondrial capacity in skeletal muscle is not stimulated by weight loss despite increases in insulin action and decreases in intramyocellular lipid content. Diabetes 57: 987-994.

15. Lowell BB, Shulman GI (2005) Mitochondrial dysfunction and type 2 diabetes. Science 307: 384-387.
Figure S2 Inhibition of mitochondrial function by Rotenone and Antimycin A. For mitochondrial metabolic inhibition, cells were treated with Rotenone $(0.1 \mu \mathrm{M})$ or Antimycin A $(10 \mu \mathrm{M})$ for $18 \mathrm{~h}$. Hepatocytes were deprived of serum for $5 \mathrm{~h}$ prior to all experimental manipulations. Cells were stained with the active mitochondrial-specific fluorescent dye, MitoTracker Orange CM- $\mathrm{H}_{2}$ TMRos, as described in Methods. Magnification is $\sim \mathrm{X} 400$.

(TIF)

Figure S3 Expression of IRS-1 under ER stress induced by tunicamycin. SK-Hepl hepatocytes were treated with vehicle or tunicamycin for $18 \mathrm{hr}$, and mRNA and protein of IRS-1 were measured by RT-PCR and immunoblotting, respectively. Phosphorylation of eIF2 $\alpha$ (peIF2 $\alpha$ ) was used for ER stress marker.

(TIF)

Table S1 Primers and PGR conditions used in this study.

(TIF)

\section{Author Contributions}

Conceived and designed the experiments: WL SP. Performed the experiments: HR SP JZ DM. Analyzed the data: WL HR SP DM JZ. Contributed reagents/materials/analysis tools: WL HR SP DM JZ. Wrote the paper: $\mathrm{WL}$.
16. Kim JA, Wei Y, Sowers JR (2008) Role of mitochondrial dysfunction in insulin resistance. Circ Res 102: 401-414.

17. Cheng Z, Guo S, Copps K, Dong X, Kollipara R, et al. (2009) Foxol integrates insulin signaling with mitochondrial function in the liver. Nature Med 15: 1307-1311.

18. Park SY, Choi GH, Choi HI, Ryu J, Jung CY, et al. (2005) Depletion of Mitochondrial DNA Causes Impaired Glucose Utilization and Insulin Resistance in L6 GLUT4myc Myocytes. J Biol Chem 280: 9855-9864.

19. Lim JH, Lee JI, Suh YH, Kim W, Song JH, et al. (2006) Mitochondrial dysfunction induces aberrant insulin signalling and glucose utilisation in murine C2C12 myotube cells. Diabetologia 49: 1924-1936.

20. Saltiel AR, Pessin JE (2002) Insulin signaling pathways in time and space. Trends Cell Biol 12: 65-71.

21. Saad MJ, Araki E, Miralpeix M, Rothenberg PL, White MF, et al. (1992) Regulation of insulin receptor substrate-1 in liver and muscle of animal models of insulin resistance. J Clin Invest 90: 1839-1849.

22. Kerouz NJ, Horsch D, Pons S, Kahn CR (1997) Differential regulation of insulin receptor substrates-1 and -2 (IRS-1 and IRS-2) and phosphatidylinositol 3-kinase isoforms in liver and muscle of the obese diabetic (ob/ob) mouse. J Clin Invest 100: 3164-3172.

23. Anai M, Ono H, Funaki M, Fukushima Y, Inukai K, et al. (1998) Different subcellular distribution and regulation of expression of insulin receptor substrate (IRS)-3 from those of IRS-1 and IRS-2. J Biol Chem 273: 29686-29692.

24. Carvalho E, Jansson PA, Nagaev I, Wenthzel AM, Smith U (2001) Insulin resistance with low cellular IRS-1 expression is also associated with low GLUT4 expression and impaired insulin-stimulated glucose transport. Faseb J 15: 1101-1103.

25. Catalano PM, Nizielski SE, Shao J, Preston L, Qiao L, et al. (2002) Downregulated IRS-1 and PPARgamma in obese women with gestational diabetes: relationship to FFA during pregnancy. Am J Physiol Endocrinol Metab 282: E522-533.

26. Brozinick JT, Jr., Roberts BR, Dohm GL (2003) Defective signaling through Akt-2 and -3 but not Akt-1 in insulin-resistant human skeletal muscle: potential role in insulin resistance. Diabetes 52: 935-941.

27. Rondinone CM, Wang LM, Lonnroth P, Wesslau C, Pierce JH, et al. (1997) Insulin receptor substrate (IRS) 1 is reduced and IRS-2 is the main docking protein for phosphatidylinositol 3-kinase in adipocytes from subjects with noninsulin-dependent diabetes mellitus. Proc Natl Acad Sci U S A 94: 4171-4175.

28. Bartel DP (2009) MicroRNAs: target recognition and regulatory functions. Cell 136: 215-233. 
29. Pillai RS, Bhattacharyya SN, Filipowicz W (2007) Repression of protein synthesis by miRNAs: how many mechanisms? Trends Cell Biol 17: 118-126.

30. Hennessy E, O'Driscoll L (2008) Molecular medicine of microRNAs: structure, function and implications for diabetes. Expert Rev Mol Med 10: e24.

31. Shi B, Sepp-Lorenzino L, Prisco M, Linsley P, deAngelis T, et al. (2007) Micro RNA 145 targets the insulin receptor substrate-1 and inhibits the growth of colon cancer cells. J Biol Chem 282: 32582-32590.

32. Zhang J, Du YY, Lin YF, Chen YT, Yang L, et al. (2008) The cell growth suppressor, mir-126, targets IRS-1. Biochem Biophys Res Commun 377: 136-140.

33. Gallagher IJ, Scheele C, Keller P, Nielsen AR, Remenyi J, et al. (2010) Integration of microRNA changes in vivo identifies novel molecular features of muscle insulin resistance in type 2 diabetes. Genome Med 2: 9.

34. Shen WF, Hu YL, Uttarwar L, Passegue E, Largman C (2008) MicroRNA-126 regulates HOXA9 by binding to the homeobox. Mol Cell Biol 28: 4609-4619.

35. Guo C, Sah JF, Beard L, Willson JK, Markowitz SD, et al. (2008) The noncoding RNA, miR-126, suppresses the growth of neoplastic cells by targeting phosphatidylinositol 3-kinase signaling and is frequently lost in colon cancers. Genes Chromosomes Cancer 47: 939-946.

36. Zeng Y, Cullen BR (2003) Sequence requirements for micro RNA processing and function in human cells. RNA 9: 112-123.
37. Butow RA, Avadhani NG (2004) Mitochondrial signaling: the retrograde response. Mol Cell 14: 1-15.

38. Chen LB (1989) Fluorescent labeling of mitochondria. Methods Cell Biol 29: $103-123$.

39. Laemmli UK (1970) Cleavage of structural proteins during the assembly of the head of bacteriophage T4. Nature 227: 680-685.

40. Amuthan G, Biswas G, Zhang SY, Klein-Szanto A, Vijayasarathy C, et al. (2001) Mitochondria-to-nucleus stress signaling induces phenotypic changes, tumor progression and cell invasion. Embo J 20: 1910-1920.

41. Kennedy ED, Maechler P, Wollheim CB (1998) Effects of depletion of mitochondrial DNA in metabolism secretion coupling in INS-1 cells. Diabetes 47: 374-380.

42. Park KS, Nam KJ, Kim JW, Lee YB, Han CY, et al. (2001) Depletion of mitochondrial DNA alters glucose metabolism in SK-Hepl cells. Am J Physiol Endocrinol Metab 280: E1007-1014.

43. Biswas G, Adebanjo OA, Freedman BD, Anandatheerthavarada HK, Vijayasarathy C, et al. (1999) Retrograde Ca2+ signaling in C2C12 skeletal myocytes in response to mitochondrial genetic and metabolic stress: a novel mode of inter-organelle crosstalk. Embo J 18: 522-533. 